\section{Application of a Deterministic Contact Model to Analyze the Contact of a Rough Surface Against a Flat Layered Surface}

\author{
H. R. Pasaribu \\ Phone: +31 53 4892476; Fax: +31 534894784 \\ e-mail: h.r.pasaribu@ctw.utwente.nl
}

\section{J. Schipper}

University of Twente, Surface Technology and Tribology, Faculty of Engineering Technology, Drienerloolaan 5, Postbus 217, 7500 AE, Enschede, The Netherlands

In this paper, a rough surface is modeled as an array of asperities represented by spheres with different radii and heights to be able to calculate the deformation (elastic, elastic-plastic, and plastic) at each asperity in contact. The total contact area and the total load carried are calculated by summarizing respectively the contact area and the load carried by each individual asperity (deterministic model). This model will diminish the assumption of an average asperity radius and enable one to calculate the contact of non-Gaussian surface more precisely. Further, in this paper, the deterministic model is used to analyze the contact behavior of a rough surface against a flat layered surface by modeling the flat layered surface as a solid that has effective mechanical properties as a function of indentation depth. [DOI: 10.1115/1.1866163]

\section{Introduction}

The work of Greenwood and Williamson [1] in studying the contact of two nominally flat surfaces in an elastic contact situation has been extended by Zhao et al. [2] by incorporating the contribution of elastic-plastic and fully plastic contact situations. These two models represent a rough surface as an array of asperities with the same radius and the asperity heights are assumed to vary randomly following the Gaussian distribution. In reality, the radius of each asperity is not the same and, in many cases, the asperity height distribution does not follow the Gaussian distribution. In this paper, a rough surface is modeled as an array of asperities represented by spheres with different radii and heights (deterministic model). Once the data of the radius and height of each single asperity is obtained, one can determine the number of asperities in contact, the contact area, and the load carried by each asperity as a function of the separation. The total contact area and the total load can be calculated by summarizing, respectively, the contact area and the load carried by each individual asperity. The present model will diminish the assumption of an average radius $[1,2]$ and enable one to calculate the contact of non-Gaussian surfaces more accurately.

Gao et al. [3] derived an analytical solution to calculate an effective Poisson's ratio of a layered surface. Further, by adopting the work of Gao et al. [3], Swain and Mecik [4] proposed a simple equation, which has been validated experimentally by them, to calculate an effective Young's modulus of a layered surface. The effective hardness of a layered surface can be calculated using an equation proposed by Bhattacharaya and Nix [5], which was obtained by fitting the finite element simulations they performed.

Contributed by the Tribology Division for publication in the ASME JOURNAL OF TRIBOLOGY. Manuscript received by the Tribology Division February 19, 2004 revised manuscript received December 21, 2004. Review conducted by: L. Chang.
Since the three mechanical properties of a layered surface $\left(v_{\text {eff }}\right.$ [3], $E_{\text {eff }}$ [4], and $H_{\text {eff }}$ [5]) can be determined as a function of indentation depth, a flat layered surface can be modeled as a solid which has an effective elasticity modulus [3], effective hardness [4], and effective Poisson ratio [5] as a function of indentation depth. This will allow one to treat the contact of a rough surface with a flat layered surface like the contact of a rough surface with a flat surface in which the mechanical properties of the flat surface are varied as a function of indentation depth, as will be discussed in the following section.

\section{Deterministic Contact Model}

Suppose $i$ numbers the asperities present at a rough surface. It is assumed in this paper that the asperities, at least near the summits, are spherical and the interactions among the neighboring contacting asperities are neglected. The asperities have different radii $\left(\beta_{\mathrm{i}}\right)$ and height $\left(z_{\mathrm{i}}\right)$ and their heights are measured from the mean height plane (see Fig. 1). When the rough surface approaches a flat surface, some asperities might have contact with the flat surface (if $z_{\mathrm{i}}>d$ ) and some might not (if $z_{\mathrm{i}}<d$ ), depending on the separation $(d)$ of the two surfaces (see Fig. 1). Assuming that the deformation solely occurs at asperity level, the local indentation depth of each asperity $\left(\omega_{i}\right)$ is determined by

$$
\omega_{\mathrm{i}}=z_{\mathrm{i}}-d
$$

Depending on the indentation depth of each asperity $\left(\omega_{\mathrm{i}}\right)$, the contact situation can be elastic, elastic-plastic, or fully plastic. The critical indentation depth $\left(\omega_{\mathrm{c} 1}\right)$ at which the contact condition changes from the elastic to the elastic-plastic contact situation (onset of yielding) was defined by Greenwood and Williamson [1] based on the work of Tabor [6] as

$$
\omega_{\mathrm{c} 1}=0.89 \beta\left(\frac{H}{E^{*}}\right)^{2}
$$

and

$$
E^{*}=\frac{E_{1} E_{2}}{E_{1}\left(1-v_{2}^{2}\right)+E_{1}\left(1-v_{1}^{2}\right)}
$$

where $\beta$ is the radius of the asperity, $H$ is the hardness, $E^{*}$ is the reduced elasticity modulus of the two surfaces in contact, $E$ is the elasticity modulus, $v$ is the Poisson ratio, and the subscripts 1 and 2 denote the two bodies in contact.

Further indentation after the onset of yielding will lead to an elastic-plastic contact situation. The range of the elastic-plastic contact area is significant and the critical indentation depth $\left(\omega_{\mathrm{c} 2}\right)$ at which the transition of the elastic-plastic to the fully plastic contact situation occurs is a few times more than the onset of yielding:

$$
\omega_{\mathrm{c} 2}=c \omega_{\mathrm{c} 1}
$$

Based on Johnson [7], Zhao et al. [2] derived that the fully plastic contact condition will occur when the indentation depth is more than 54 times the onset of the first yielding $(c=54)$. According to Kogut and Etsion [8], based on the finite element simulations performed by them, constant $c$ in Eq. (4) can be about 110 . Indeed, a different value for the constant $c$ will certainly lead to a significant difference in calculating the contact area and the load carried by the asperities. Recently, Jackson and Green [9] proposed a new equation in calculating the contact area of a sphere pressed against a rigid flat surface. The proposed equation is a fit based on the finite element simulations they performed. Depending on the penetration depth, the contact area calculated by using the model of Jackson and Green [9] shows about a $0 \%$ to $20 \%$ difference from the contact area calculated using the model of Zhao et al. [2]. However, Jackson and Green [9] also reported in their paper that they observed an error up to $17 \%$ between the contact area obtained from their finite element simulation and the analytical Hertzian solution. This error is in the range of differ- 


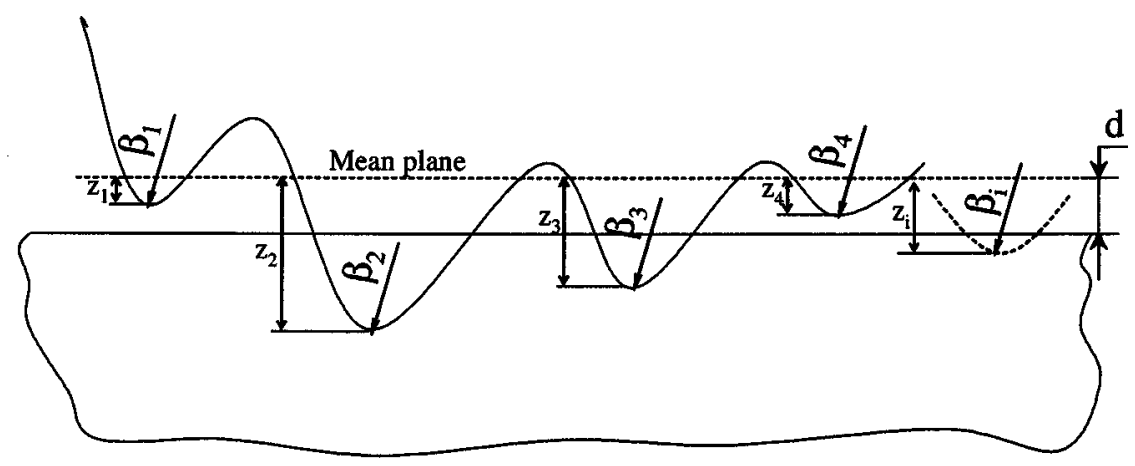

Fig. 1 Contact model of a rough surface with a flat surface

ence between the contact area calculated with the model of Zhao et al. [2] and the contact area calculated with the model of Jackson and Green [9]. Therefore, in this paper, with the abovementioned reason and simplicity reason, the calculation of the contact area is carried out based on the work of Zhao et al. [2].

The contact area of each asperity $\left(A_{i}\right)$ at elastic $\left(A_{\mathrm{ie}}\right)$, elasticplastic $\left(A_{\text {iep }}\right)$ or fully plastic $\left(A_{\text {ip }}\right)$ contact situation can be calculated as

$$
A_{\mathrm{i}}\left(\omega_{\mathrm{i}}\right)= \begin{cases}A_{\text {ie }}\left(\omega_{\mathrm{i}}\right) & \text { if } \omega_{\mathrm{i}}<\omega_{\mathrm{c} 1}\left(\omega_{\mathrm{i}}\right) \\ A_{\text {iep }}\left(\omega_{\mathrm{i}}\right) & \text { if } \omega_{\mathrm{c} 1}\left(\omega_{\mathrm{i}}\right)<\omega_{\mathrm{i}}<\omega_{\mathrm{c} 2}\left(\omega_{\mathrm{i}}\right) \\ A_{\text {ip }}\left(\omega_{\mathrm{i}}\right) & \text { if } \omega_{\mathrm{c} 2}\left(\omega_{\mathrm{i}}\right)<\omega_{\mathrm{i}}\end{cases}
$$

where

$$
\begin{gathered}
A_{\mathrm{ie}}\left(\omega_{\mathrm{i}}\right)=\pi \beta_{\mathrm{i}} \omega_{\mathrm{i}} \\
A_{\text {iep }}\left(\omega_{\mathrm{i}}\right)=\pi \beta \omega_{\mathrm{i}}\left[1-2\left(\frac{\omega_{\mathrm{i}}-\omega_{\mathrm{c} 1}}{\omega_{\mathrm{c} 2}-\omega_{\mathrm{c} 1}}\right)^{3}+3\left(\frac{\omega_{\mathrm{i}}-\omega_{\mathrm{c} 1}}{\omega_{\mathrm{c} 2}-\omega_{\mathrm{c} 1}}\right)^{2}\right] \\
A_{\mathrm{ip}}\left(\omega_{\mathrm{i}}\right)=2 \pi \beta_{\mathrm{i}} \omega_{\mathrm{i}}
\end{gathered}
$$

The load carried by each asperity $\left(P_{\mathrm{i}}\right)$ at elastic $\left(P_{\mathrm{ie}}\right)$, elasticplastic $\left(P_{\text {iep }}\right)$ of fully plastic $\left(P_{\text {ip }}\right)$ contact situation can be calculated as:

$$
P_{\mathrm{i}}\left(\omega_{\mathrm{i}}\right)= \begin{cases}P_{\mathrm{ie}}\left(\omega_{\mathrm{i}}\right) & \text { if } \omega_{\mathrm{i}}<\omega_{\mathrm{c} 1}\left(\omega_{\mathrm{i}}\right) \\ P_{\text {iep }}\left(\omega_{\mathrm{i}}\right) & \text { if } \omega_{\mathrm{c} 1}\left(\omega_{\mathrm{i}}\right)<\omega_{\mathrm{i}}<\omega_{\mathrm{c} 2}\left(\omega_{\mathrm{i}}\right) \\ P_{\mathrm{ip}}\left(\omega_{\mathrm{i}}\right) & \text { if } \omega_{\mathrm{c} 2}\left(\omega_{\mathrm{i}}\right)<\omega_{\mathrm{i}}\end{cases}
$$

where

$$
\begin{gathered}
P_{\mathrm{ie}}=\frac{4}{3} E^{*} \beta_{\mathrm{i}}^{0.5} \omega_{\mathrm{i}}^{1.5} \\
P_{\mathrm{iep}}=\left[H-0.6 H \frac{\ln \omega_{\mathrm{c} 2}-\ln \omega_{\mathrm{i}}}{\ln \omega_{\mathrm{c} 2}-\ln \omega_{\mathrm{c} 1}}\right] A_{\mathrm{iep}} \\
P_{\mathrm{ip}}=H A_{\mathrm{ip}}
\end{gathered}
$$

The total contact area $\left(A_{\text {tot }}\right)$ and the total load carried $\left(P_{\text {tot }}\right)$ can be calculated by summing up the individual contact areas and the loads carried by each individual asperity:

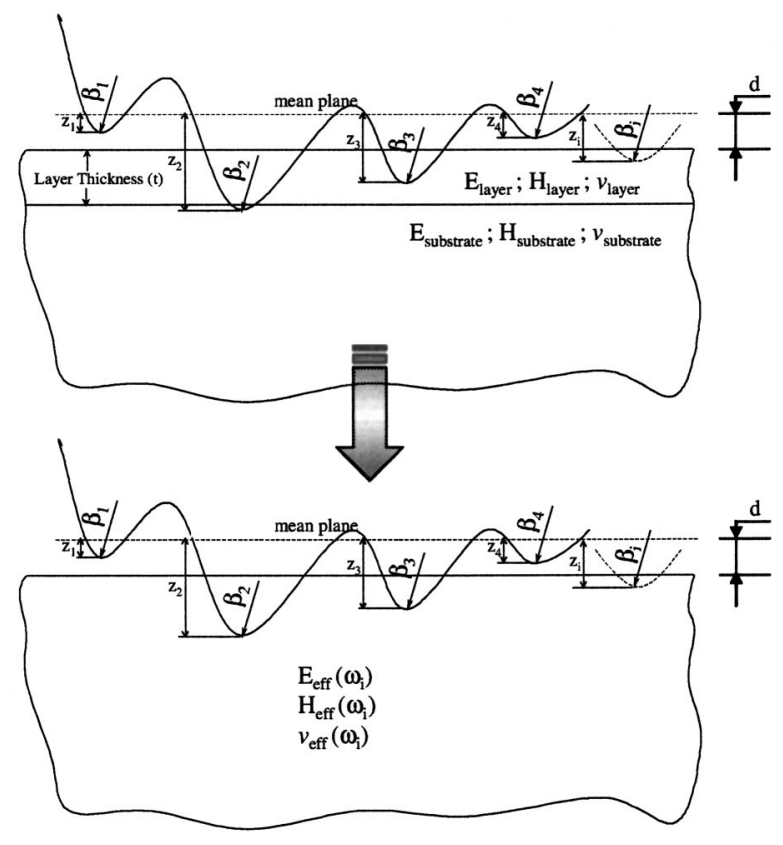

Fig. 2 Contact model of a rough surface with a flat layered surface

$$
A_{\text {tot }}=\sum_{\mathrm{i}} A_{\mathrm{i}} ; \quad P_{\mathrm{tot}}=\sum_{\mathrm{i}} P_{\mathrm{i}}
$$

\section{Contact of a Rough Surface With a Flat Layered Surface}

In this paper, a layered surface is modeled like a solid that has effective mechanical properties as a function of indentation depth by assuming that the layer is perfectly bounded to the substrate (see Fig. 2). The effective mechanical properties of a layered surface vary as a function of indentation depth and the values of these properties range between the value of the layer itself and of the substrate.

The effective Poisson's ratio $\left(v_{\text {eff }}\right)$ [3], the effective elasticity modulus $\left(E_{\text {eff }}\right)[4]$, and the effective hardness $\left(H_{\text {eff }}\right)[4]$ of a layered surface can be calculated respectively as follows:

$$
\begin{gathered}
v_{\text {eff }}\left(\omega_{\mathrm{i}}\right)=v_{\mathrm{s}}+\left(v_{1}-v_{\mathrm{s}}\right) I_{1}\left(\xi\left(\omega_{\mathrm{i}}\right)\right) \\
E_{\mathrm{eff}}\left(\omega_{\mathrm{i}}\right)=E_{\mathrm{s}}+\left(E_{1}-E_{\mathrm{s}}\right) I_{0}\left(\xi\left(\omega_{\mathrm{i}}\right)\right)
\end{gathered}
$$




$$
H_{\mathrm{eff}}\left(\omega_{\mathrm{i}}\right)=\left\{\begin{array}{l}
H_{\mathrm{s}}+\left(H_{1}-H_{\mathrm{s}}\right) \exp \left(-\frac{\sigma_{\mathrm{yl}} E_{\mathrm{s}}}{\sigma_{\mathrm{ys}} E_{1}}\left(\frac{\omega_{\mathrm{i}}}{\mathrm{t}}\right)^{2}\right) \text { if } H_{\mathrm{s}}>H_{1} \\
H_{\mathrm{s}}+\left(H_{1}-H_{\mathrm{s}}\right) \exp \left(-\frac{\left(H_{1} / H_{\mathrm{s}}\right)}{\left(\sigma_{\mathrm{yl}} / \sigma_{\mathrm{ys}}\right)\left(E_{\mathrm{yl}} / E_{\mathrm{ys}}\right)^{0.5}}\left(\frac{\omega_{\mathrm{i}}}{\mathrm{t}}\right)\right) \quad \text { if } H_{1}>H_{\mathrm{s}}
\end{array}\right.
$$

with

$$
\begin{aligned}
I_{1}\left(\xi\left(\omega_{\mathrm{i}}\right)\right)= & \frac{2}{\pi} \tan ^{-1}\left(\xi\left(\omega_{\mathrm{i}}\right)\right)+\frac{\xi\left(\omega_{\mathrm{i}}\right)}{\pi} \ln \frac{1+\xi\left(\omega_{\mathrm{i}}\right)^{2}}{\xi(\omega)^{2}} \\
I_{0}\left(\xi\left(\omega_{\mathrm{i}}\right)\right)= & \frac{2}{\pi} \tan ^{-1}\left(\xi\left(\omega_{\mathrm{i}}\right)\right)+\frac{1}{2 \pi\left(1-v_{\text {eff }}\right)}[(1 \\
& \left.\left.-2 v_{\text {eff }}\right) \xi\left(\omega_{\mathrm{i}}\right) \ln \frac{1+\xi\left(\omega_{\mathrm{i}}\right)^{2}}{\xi\left(\omega_{\mathrm{i}}\right)^{2}}-\frac{\xi\left(\omega_{\mathrm{i}}\right)}{1+\xi\left(\omega_{\mathrm{i}}\right)^{2}}\right]
\end{aligned}
$$

and

$$
\begin{gathered}
\xi\left(\omega_{\mathrm{i}}\right)=\frac{t}{a\left(\omega_{\mathrm{i}}\right)} \\
a\left(\omega_{\mathrm{i}}\right)=\beta^{0.5} \omega_{\mathrm{i}}^{0.5}
\end{gathered}
$$

where $H$ is the hardness, $E$ is the Young's modulus, $\sigma_{\mathrm{y}}$ is the yield stress of the material, $\omega$ is the indentation depth, $t$ is the layer thickness, and subscript $\mathrm{s}$ and 1 refer to substrate and layer, respectively.

By inserting the three mechanical properties of the layered surface ( $v_{\text {eff }}, E_{\text {eff }}$, and $\left.H_{\text {eff }}\right)$ into Eqs. (5) and (9) ( $v_{2}$ replaced by $v_{\text {eff }}, E_{2}$ replaced by $E_{\text {eff }}$, and $H_{2}$ replaced by $H_{\text {eff }}$ ), one can calculate the contact of a rough surface against a flat layered surface.

\section{Results and Discussions}

4.1 Contact of a Rough Surface With a Flat Surface. To compare the statistical model of Zhao et al. [2] with the deterministic model (present model), two different surfaces are generated with surface parameters as shown in Table 1 . These surfaces consist of asperities with different radii $\left(\beta_{\mathrm{i}}\right)$ and the heights $\left(z_{\mathrm{i}}\right)$ vary from a mean height plane (see Fig. 3). The radius and the heights of each asperity are determined based on the work of Greenwood [10] using a five-point summit. Suppose these two rough surfaces $(E=210 \mathrm{GPa}, H=7.6 \mathrm{GPa}, v=0.3)$ are brought into contact with a flat surface $(E=210 \mathrm{GPa}, H=7.6 \mathrm{GPa}, v=0.3$, respectively). The dimensionless separation $(d / \sigma)$ as a function of dimensionless load $\left(P_{\text {tot }} /\left(A_{\text {nom }} E^{*}\right)\right)$ calculated by the present model and the model of Zhao et al. [2] are presented in Fig. 4.

Table 1 Surface parameters of the two surfaces used

\begin{tabular}{|c|c|c|c|}
\hline Case & $\beta_{\text {avg }}[\mathrm{m}]$ & $\sigma[\mathrm{m}]$ & $\eta\left[\mathrm{m}^{-2}\right]$ \\
\hline 1 & $2 \times 10^{-5}$ & $4.8 \times 10^{-8}$ & $6 \times 10^{10}$ \\
\hline 2 & $1.6 \times 10^{-6}$ & $2.6 \times 10^{-7}$ & $9 \times 10^{10}$ \\
\hline
\end{tabular}
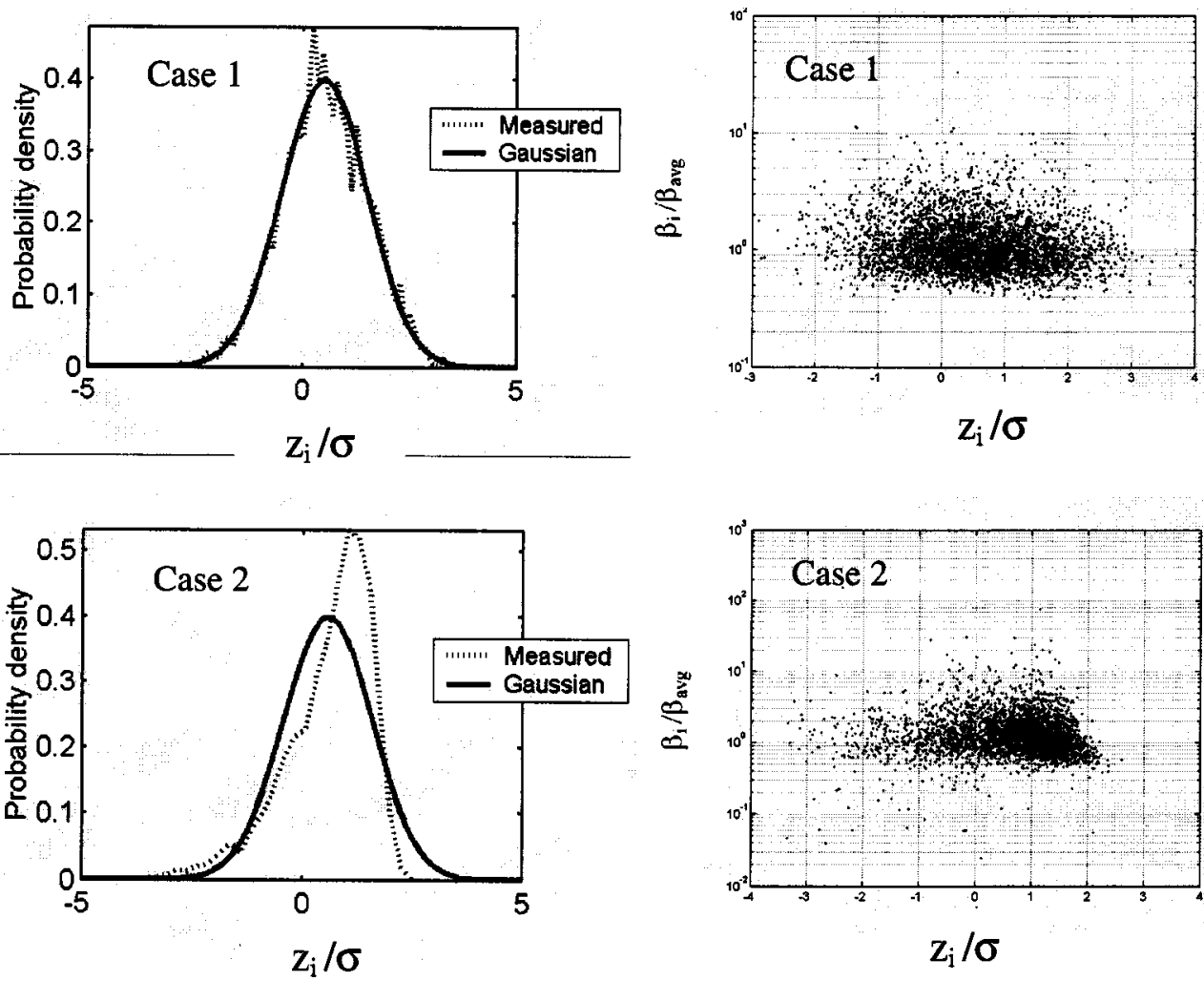

Fig. 3 The asperity height distribution and radius distribution of the two surfaces, i.e., radius ratio $\left(\boldsymbol{\beta}_{i} / \boldsymbol{\beta}_{\mathrm{avg}}\right)$ as a function of normalized height $\left(z_{i} / \sigma_{s}\right)$ 


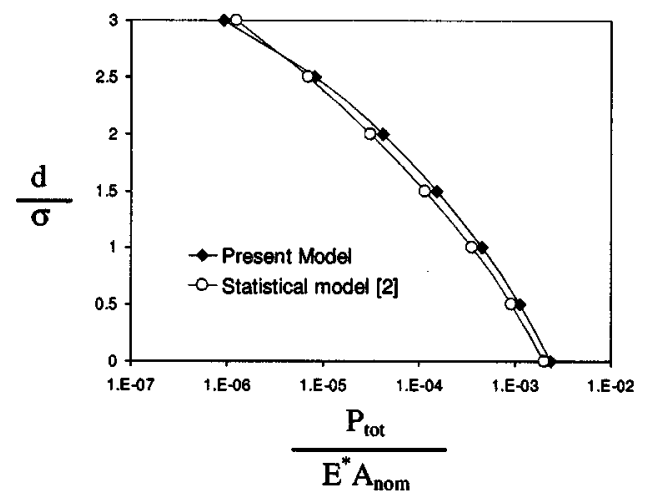

(a)

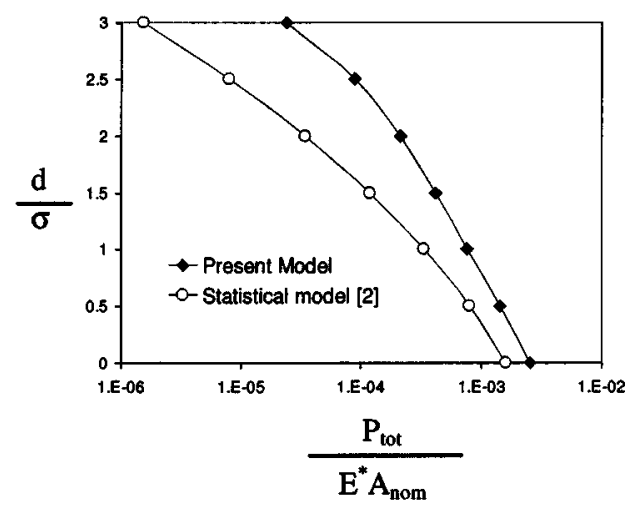

(b)

Fig. 4 Comparison of the deterministic model and the statistical model for the two surfaces in contact with a flat surface (without layer) (a) case 1 and (b) case 2 (see Table 1)

It is clear from Fig. 4 that for the two cases studied in this paper, the results obtained with the deterministic model and the statistical model show a significant difference and for the nonGaussian surface (case 2) the difference in calculating the separation is more pronounced. This is due to the fact that the asperities have different radii (see Fig. 3), each asperity has its own transition from elastic to plastic contact conditions and, by this, obviously for the same indentation depth, the load carried and the contact area calculated by the statistical model and the deterministic model will reveal different values.

4.2 Contact of a Sphere With a Flat Layered Surface. The present model, where the flat layered surface is modeled as a solid with effective mechanical properties, is used to simulate the experimental conditions performed by Sherbiney [11] and the numerical simulation (finite element method) performed by Tang and Arnell [12]. Sherbiney [11] performed an experiment on a steel ball $(\beta=12.75 \mathrm{~mm}, E=210 \mathrm{GPa}, H=7.6 \mathrm{GPa}, v=0.3)$ pressed against a flat lead layer $(t=1.2 \mu \mathrm{m}, E=18 \mathrm{GPa}, H$ $=0.17 \mathrm{GPa}, v=0.35)$ deposited on a mild steel substrate $(E$ $=140 \mathrm{GPa}, H=1.86 \mathrm{GPa}, v=0.3)$. Tang and Arnell [10] performed finite element simulations of a diamond indenter $(\beta=100$ $\mu \mathrm{m}, E=1140 \mathrm{GPa}, v=0.07)$ pressed against a ceramic layer $(t$ $=1 \mu \mathrm{m}, E=400 \mathrm{GPa}, H=21 \mathrm{GPa}, v=0.3)$ on a steel substrate $(E=200 \mathrm{GPa}, H=7.6 \mathrm{GPa}, v=0.07)$.

In Fig. 5, the normal approach as a function of load for the case of Sherbiney [11] and the normal load as a function of indentation depth according to Arnell and Tang [12] are calculated with the present model and the results are plotted together with the experimental data [11] and the numerical simulation results [12] obtained by them.

Figure 5 clearly shows that the present model is in good agreement with the experimental results reported by Sherbiney [11] and the finite element simulation presented by Tang and Arnell [12]. Therefore, this simple model of a sphere in contact with a flat layered surface can be extended to the contact of a rough surface against a flat layered surface, assuming that the interactions between the neighboring asperities are negligible.

4.3 Contact of a Rough Surface With a Flat Layered Surface. Suppose a flat surface $(E=210 \mathrm{GPa}, H=7.6 \mathrm{GPa}, v$ $=0.3)$ is covered with lead layer of varying thickness $(E$ $=18 \mathrm{GPa}, H=0.17 \mathrm{GPa}, v=0.35$ ). If this flat layered surface is brought into contact with the two rough surfaces listed in Table 1 $(E=210 \mathrm{GPa}, H=7.6 \mathrm{GPa}, v=0.3)$, the dimensionless separation $(d / \sigma)$ and the dimensionless contact area $\left(A_{t} / A_{\text {nom }}\right)$ as a function of dimensionless load $\left[P_{\text {tot }} /\left(E_{t=0}^{*} A_{\text {nom }}\right)\right]$ for these two cases are shown in Fig. 6.

As shown in Fig. 6, when the flat surface is coated by a lead layer with certain thickness, the value of the dimensionless separation as a function of the dimensionless load of the layered system is between the value of the system without a layer and the

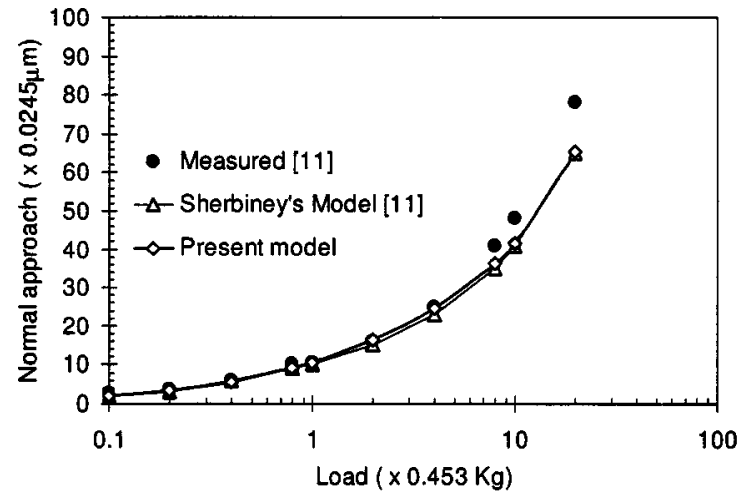

(a)

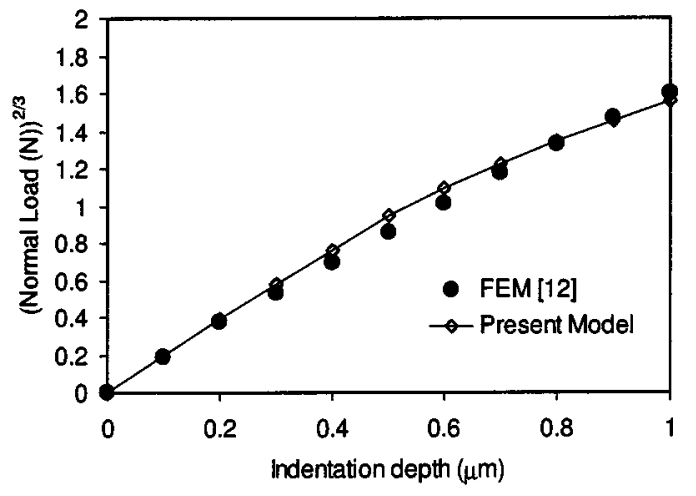

(b)

Fig. 5 The comparison between the measurements performed by Sherbiney [11] (a) and the FEM simulation carried out by Tang and Arnell [12] (b) with the calculated results using the present model. (The units along the axis are according to the literature data $[11,12]$. 

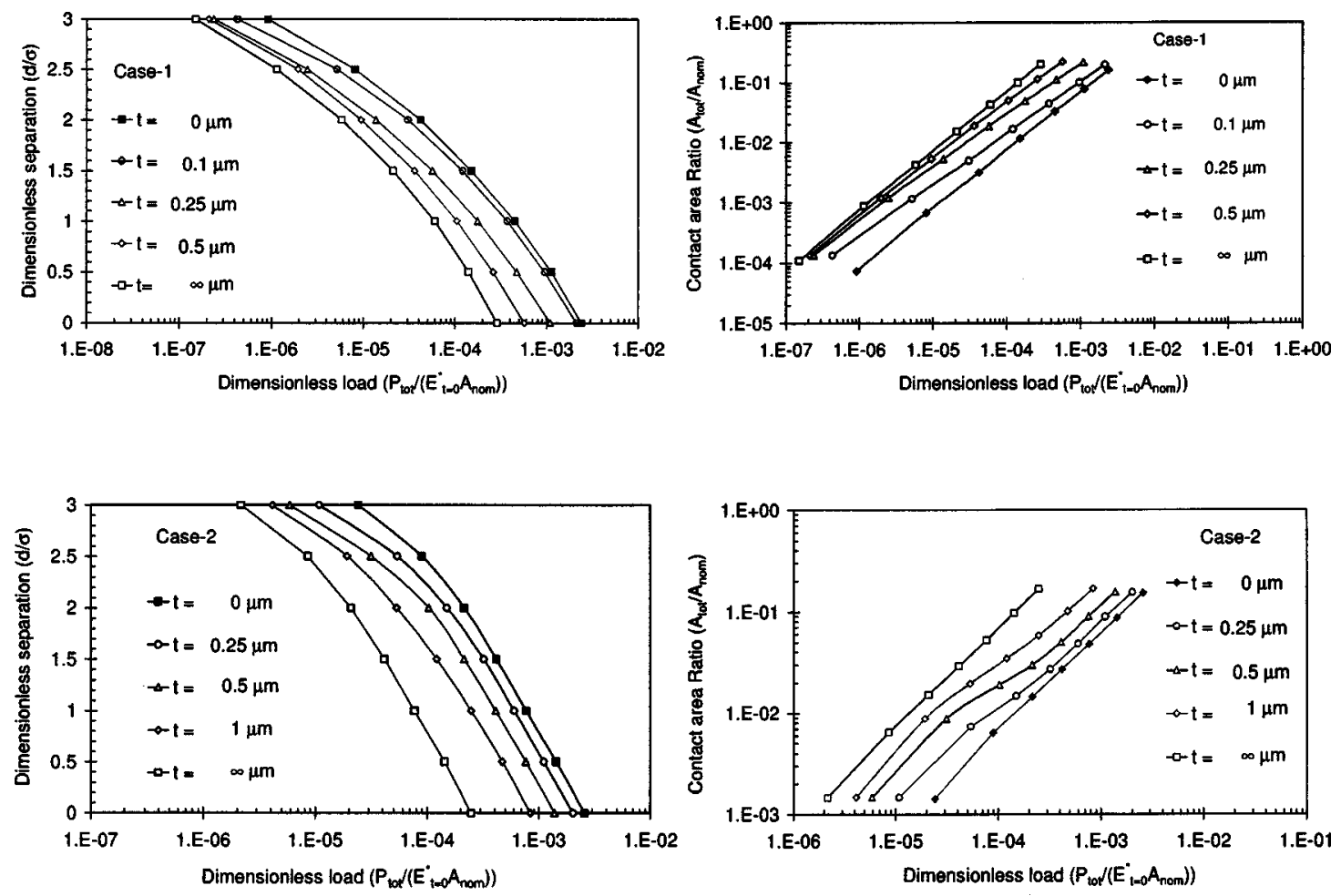

Fig. 6 Dimensionless separation $(d / \sigma)$ and dimensionless contact area $\left(A_{t} / A_{\text {nom }}\right)$ as a function of dimensionless load $\left(P_{\text {tot }} /\left(E_{t=0}^{*} A_{\text {nom }}\right)\right)$ for different thicknesses $(t)$ of the lead layer on a flat surface in contact with the surfaces, case 1 and case 2 (see Table 1)

system with a very thick layer $(t=\infty)$. These results also show that the effect of the substrate is less as the penetration of the asperities into the layer is less as well.

\section{Conclusions}

A deterministic contact model of a rough surface against a flat layered surface has been presented. As for the case of a sphere pressed against a flat layered surface (single asperity model), the proposed model shows good agreement with the experimental and numerical results, and one can extend the single asperity model to calculate the contact of a rough surface with a flat layered surface by analyzing the contact of each individual asperity (deterministic model). The deterministic contact model allows one to analyze the contact of non-Gaussian surfaces and diminish the assumption of an average asperity radius, which makes the calculation of the contact area and the load carried more accurate. The same method can be applied to ellipsoids $[13,14]$ instead of spheres.

\section{References}

[1] Greenwood, J. A., and Williamson, J. B. P., 1966, "Contact of Nominally Flat Surface," Proc. R. Soc. London, Ser. A, 295, pp. 300-319.

[2] Zhao, Y., Maietta, D. M., and Chang, L., 2000, "An Asperity Microcontact Model Incorporating the Transition From Elastic Deformation to Fully Plastic Flow," ASME J. Tribol., 122, pp. 86-93.
[3] Gao, H., Chui, C., and Lee, J., 1992, "Elastic Contact Versus Indentation Model of Multi-Layered Material," Int. J. Solids Struct., 29, pp. 2471-2492.

[4] Swain, M. V., and Mencik, J., 1994, "Mechanical Characterization of Thin Films Using Spherical Tipped Indenters," Thin Solid Films, 253, pp. 204-211.

[5] Bhattacharaya, A. K., and Nix, W. D., 1988, "Analysis of Elastic and Plastic Deformation Associated With Indentation Testing of Thin Films on Substrates," Int. J. Solids Struct., 24, pp. 1287-1298.

[6] Tabor, D., 1951, The Hardness of Metals, Oxford University Press, Oxford.

[7] Johnson, K. L., 1995, Contact Mechanics, Cambridge University Press, Cambridge.

[8] Kogut, L., and Etsion, I., 2002, "Elastic-Plastic Contact Analysis of a Sphere and a Rigid Flat,” Trans. ASME, J. Appl. Mech., 69(5), pp. 657-662.

[9] Jackson, R. L., and Green, I., 2003, "A Finite Element Study of Elasto-Plastic Hemispherical Contact," Proc. of 2003 STLE/ASME Joint Tribology Conference, 2003TRIB-268.

[10] Greenwood, J. A., 1984, “A Unified Theory of Surface Roughness,” Proc. R. Soc. London, Ser. A, 393, pp. 133-157.

[11] Sherbiney, M. G. D., 1975, "Tribological Properties of Ion-Plated ThinMetallic Films," Ph.D. thesis, University of Salford, England.

[12] Tang, K. C., and Arnell, R. D., 1999, "Determination of Coating Mechanical Properties Using Spherical Indenters," Thin Solid Films, 355-356, pp. 263269.

[13] Bush, A. W., Gibson, R. D., and Thomas, T. R., 1975, "Elastic Contact of a Rough Surface," Wear, 35, pp. 87-111.

[14] Jeng, Y. R., and Wang, P. Y., 2003, "An Elliptical Microcontact Model Considering Elastic, Elastoplastic, and Plastic Deformation," ASME J. Tribol., 125, pp. 232-240. 